\title{
Elasticity of substitution and growth: normalized CES in the diamond model ${ }^{\star}$
}

\author{
Kaz Miyagiwa $^{1}$, Chris Papageorgiou ${ }^{2}$ \\ 1 Department of Economics, Emory University, Atlanta, GA 30322, USA \\ 2 Department of Economics, Louisiana State University, Baton Rouge, LA 70803-6306, USA \\ (e-mail: cpapa@1su.edu)
}

Received: October 27, 2001; revised version: February 25, 2002

Summary. It is often asserted that the more substitutable capital and labor are in the aggregate production the more rapidly an economy grows. Recently this has been formally confirmed within the Solow model by Klump and de La Grandville (2000). This paper demonstrates that there exists no such monotonic relationship between factor substitutability and growth in the Diamond overlapping-generations model. In particular, we prove that, if capital and labor are relatively substitutable, a country with a greater elasticity of substitution exhibits lower per capita output growth in transit and in steady state.

Keywords and Phrases: CES, Diamond overlapping generations model, Economic growth.

JEL Classification Numbers: E13, E23, O40.

\section{Introduction}

The development of modern growth theory owes much to the use of Cobb-Douglas aggregate production function. However, it is also true that the general conclusions of growth theory have been somewhat limited because of the unitary elasticity of substitution between capital and labor implied by the Cobb-Douglas production function. This observation has led Pitchford (1960) to use the constant-elasticityof-substitution (CES) production function in the neoclassical growth model and

\footnotetext{
* We thank Rainer Klump, Theodore Palivos and an anonymous referee for their valuable comments. Correspondence to: C. Papageorgiou
} 
investigate the effect on growth of the elasticity of substitution itself. ${ }^{1}$ Among his findings is the fact that an increase in the elasticity of substitution not only increases the curvature of the isoquant for a given level of output but also causes the isoquant to shift inwards by making labor and capital more efficient. The latter effect has the implication that despite initially having identical endowments of capital and labor an economy with a higher elasticity of substitution begins its growth path at a greater level of per-capita income than an economy with a smaller elasticity of substitution.

Recently, Klump and de La Grandville (2000) (henceforth KG) have used a certain normalization procedure to correct the above bias inherent in the use of CES production function. Using their "normalized" CES production function in the Solow growth model they have found that a country exhibiting a higher elasticity of substitution experiences greater capital and output per worker both in transition and in steady state. The objective of this paper is to extend this line of research within the Diamond (1965) overlapping-generations model. We believe it is a worthwhile extension since the Diamond model has increasingly been used in recent years to study economic growth as an alternative to Solow model.

Our main conclusion is that the Diamond model does not exhibit the monotonic relationship between the elasticity of substitution and capital and output per worker either in transition or in steady state. More specifically, we show that, if the elasticity of substitution is sufficiently high, a further increase in the elasticity of substitution lowers output and income per worker both in transition and in steady state. Our result obtains because unlike in the Solow model, agents save a fraction of wage income in the Diamond model. As shown by KG, a higher elasticity implies an increase in total income but also a fall in labor share. In a model where agents save out of wage income, this makes it ambiguous how savings will be affected by an increase in the elasticity of substitution. We show that if the elasticity of substitution exceeds one by enough higher elasticities imply lower growth and a lower steady state capital stock.

\section{The normalized CES production function in the Solow model}

The normalization procedure proposed by KG is as follows. Given the standard intensive-form CES production function $f\left(k_{t}\right)=A\left[\delta k_{t}^{\rho}+(1-\delta)\right]^{\frac{1}{\rho}}$, where $k_{t}$ is the capital per worker at time $t$, choose arbitrary baseline values for capital per worker $(\bar{k})$, output per worker $(\bar{y})$ and the marginal rate of substitution between capital and labor defined by $\bar{m}=\left[f(\bar{k})-\bar{k} f^{\prime}(\bar{k})\right] / f^{\prime}(\bar{k})$ (primes denote derivatives). Then, use those baseline values to solve for the normalized efficiency parameter $A(\sigma)=$ $\bar{y}\left(\frac{\bar{k}^{1-\rho}+\bar{m}}{\bar{k}+\bar{m}}\right)^{1 / \rho}$, and the normalized distribution parameter $\delta(\sigma)=\frac{\bar{k}^{1-\rho}}{k^{1-\rho}+\bar{m}}$ as a function of $\sigma=\frac{1}{1-\rho}$, the elasticity of substitution. Substituting these normalized

\footnotetext{
1 There are two types of CES functions: the homothetic CES pioneered by Arrow et al. (1961), and the nonhomothetic CES derived by Sato (1975) and Shimomura (1999). This paper considers only homothetic CES.
} 


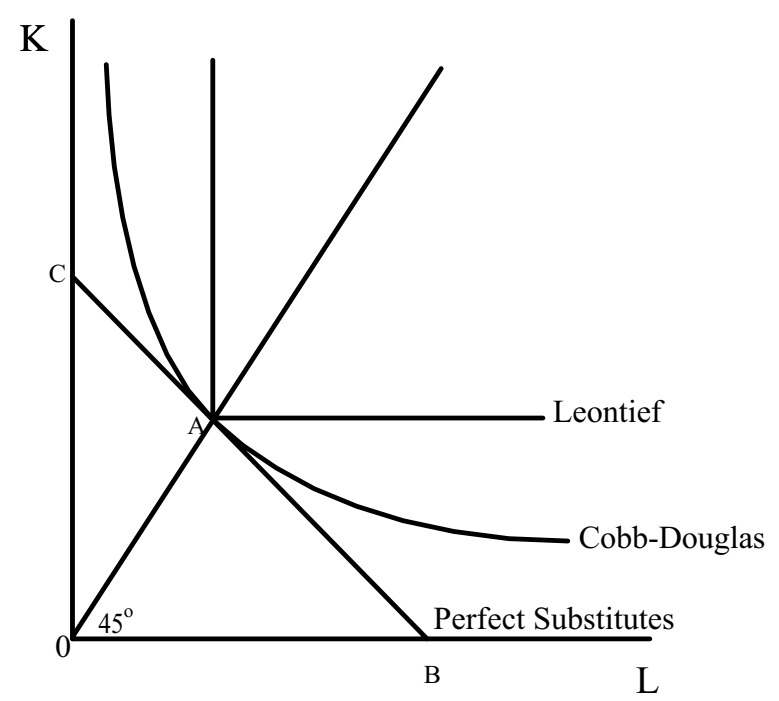

Figure 1. Illustration of de La Grandville's normalized CES production function

parameters into the initial equation yields the normalized CES production function: ${ }^{2}$

$$
f_{\sigma}\left(k_{t}\right)=A(\sigma)\left\{\delta(\sigma) k_{t}^{\rho}+[1-\delta(\sigma)]\right\}^{\frac{1}{\rho}} .
$$

Figure 1 illustrates the result of this normalization. Despite disparate values for $\sigma(0,1,+\infty)$, all the isoquants for the baseline level of output are shown to go through the common point (point $A$ ) defined by $\bar{k}$ (given by ray $O A$ ) and $\bar{m}$ (given by line $B A C$ ).

The normalization generates a family of dynamical paths in the Solow growth model that depend only on the value of $\sigma$. Paths of capital per worker for three values of $\sigma$ are shown in Figure 2. Figure 2 differs from Figure 1 in KG (2000, p. 284) because here the Solow model is recast in a discrete-time setting to facilitate comparison with the Diamond model below. More specifically, the paths shown in the figure are generated by the dynamical equation

$$
k_{t+1}=\frac{\gamma}{1+n} f_{\sigma}\left(k_{t}\right)
$$

where $\gamma$ is the exogenous saving rate out of output per worker, $n$ is the exogenous labor growth rate and where for simplicity capital is assumed to depreciate fully at the end of each period. ${ }^{3}$

Despite the translation into the discrete-time setting, the KG result is evident; a country having a greater value of $\sigma$ clearly has more capital per worker in transition

\footnotetext{
${ }^{2}$ For extensive discussions on the normalized CES function see Klump and Preissler (2000, pp. 44 45).

${ }^{3}$ In constructing Figures 2, 3, 4, we set $\gamma=0.5, n=0.01$. Parametric examples of the dynamic relationship between $y_{t+1}$ and $y_{t}$ are available upon request.
} 


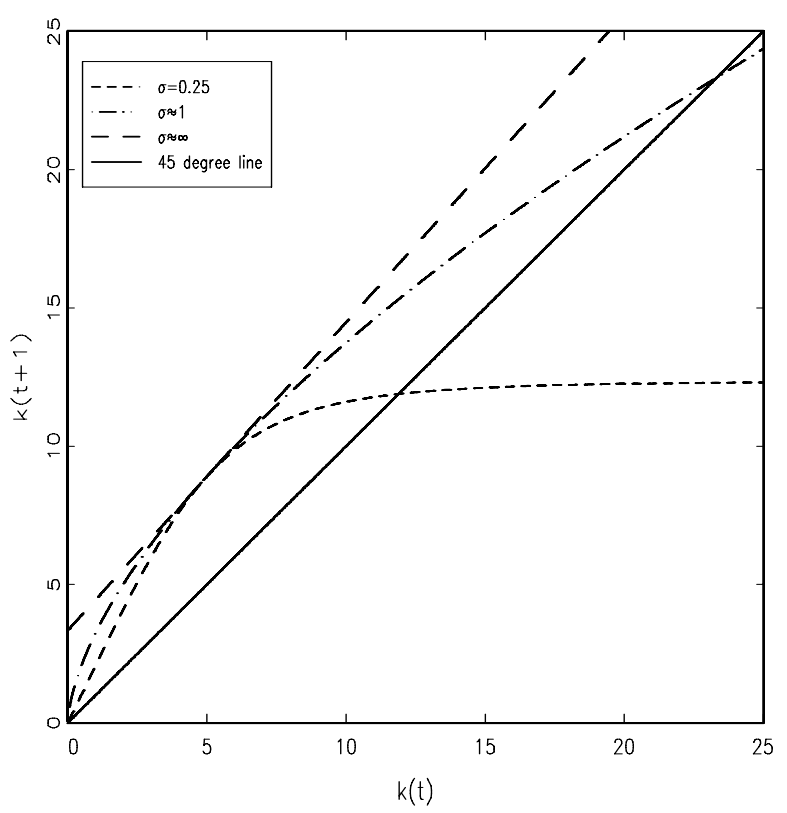

Figure 2. Transitional paths of per capita capital for different $\sigma$ in the Solow model

and in steady state than a country endowed with a lower value of $\sigma$. It follows that, the greater the value of $\sigma$, the greater is income per worker both in transition and in steady state.

\section{The normalized CES production function in the diamond model}

In the Diamond (1965) overlapping-generations model a new generation is born at the beginning of every period. Agents are identical and live for two periods. In the first period each agent supplies a unit of labor inelastically and receives a competitive wage

$$
w_{\sigma, t}=f_{\sigma}\left(k_{t}\right)-k_{t} f_{\sigma}^{\prime}\left(k_{t}\right)=[1-\delta(\sigma)][A(\sigma)]^{\rho}\left[f_{\sigma}\left(k_{t}\right)\right]^{1-\rho} .
$$

To make the model consistent with the Solow model, assume that agents save a fixed proportion $\gamma$ of the wage income to finance consumption in the second period of their lives. All savings are invested as capital to be used in the next period's production; that is

$$
k_{t+1}=\frac{\gamma}{1+n} w_{\sigma}\left(k_{t}\right)=\frac{\gamma}{1+n}[1-\delta(\sigma)][A(\sigma)]^{\rho}\left[f_{\sigma}\left(k_{t}\right)\right]^{1-\rho} \equiv h_{\sigma}\left(k_{t}\right),
$$

where $n$ is the exogenous labor growth rate and where capital depreciates fully. ${ }^{4}$ Equation (2) determines the dynamical path of capital per worker. Then, the dynamical path of output per worker is obtained from (1).

\footnotetext{
4 Alternatively, we could assume that agents have preferences over consumption in the two periods of their lives given by $U\left(c_{t}^{1}, c_{t+1}^{2}\right)=(1-\gamma) \ln c_{t}^{1}+\gamma \ln c_{t+1}^{2}$, where $c_{t+j}^{i}$ is period $i$ consumption
} 
Steady states for $k$ (denoted by $*$ ) are solutions to the polynomial equation

$$
k-h_{\sigma}(k)=0 \text {. }
$$

If $\sigma \geq 1(\rho \in[0,1])$, there always exists one unique positive steady state for $k^{*}$, since $\lim _{k \rightarrow 0} h_{\sigma}^{\prime}(k)>1$ and $\lim _{k \rightarrow+\infty} h_{\sigma}^{\prime}(k)=0$. If $\sigma<1(\rho<0)$, there are either zero or two positive and distinct steady-state values for $k^{*}$, depending on the value of the scale factor $A(\sigma) .^{5}$

We now turn to our two main findings. (All proofs are in the Appendix.)

Theorem 1 Suppose that a country is represented by the one-sector Diamond model with a normalized CES aggregate production function. If $\rho \geq \frac{\bar{m}}{\bar{k}}$, for any $k_{t}>\bar{k}$

(A) the higher the elasticity of substitution the lower the level of capital and output per worker at any stage of the transition path, and

(B) the higher the elasticity of substitution the lower the growth rates of capital and output per worker along the transitional path.

Theorem 2 Suppose that a stable steady state exists in the one-sector Diamond model with a normalized CES aggregate production function. If $\rho \geq \frac{\bar{m}}{\bar{k}}$, the higher the elasticity of substitution, the lower the steady-state level of capital and output per worker.

Note that $\rho \geq \frac{\bar{m}}{\bar{k}}$ implies $\sigma \geq \frac{\bar{k}}{\bar{k}-\bar{m}}>1$.

Figure 3 illustrates the dynamical paths of capital per worker in the Diamond model for $\rho \geq \frac{\bar{m}}{k}$, where we set $\bar{m}=1$ and $\bar{k}=5$. As $\sigma$ increases from 1.25 to 5 and to $\infty$, the level of capital per worker falls both in transition and in steady state for any $k_{t}>\bar{k} .^{6}$

Moreover, if $\rho<\frac{\bar{m}}{k}$ the relationship between $\sigma$ and the level of capital per worker is not unique because, as shown in Figure 4, the dynamical paths of $k$ for different values of $\sigma$ cross each other at some $k_{t}>\bar{k} .^{7}$

Why do our results contrast with those from the Solow model? The Diamond model differs from the Solow model in one important respect: individual savings

by the representative agent in period $t+j, j=0,1$. The representative agent maximizes $U\left(c_{t}^{1}, c_{t+1}^{2}\right)$ subject to the constraint, $c_{t}^{1}+\frac{c_{t+1}^{2}}{R_{\sigma, t+1}} \leq w_{\sigma, t}$, where $w_{\sigma, t}$ and $R_{\sigma, t+1}$ represent the returns to labor and capital, respectively. Maximization yields the transition equation, $k_{t+1}=\frac{\gamma}{1+n} w_{\sigma}\left(k_{t}\right)$, which is equivalent to equation (2).

5 When there are two positive steady states, the larger of the two is locally asymptotically stable. In this case, the trivial steady state $\left(k^{*}=0\right)$ is also locally asymptotically stable. The domains of attraction of the two stable steady states are distinct, and depend on whether the initial capital stock lies above or below the locally unstable equilibrium. The conditions for and characterization of multiple equilibria in the Diamond (1965) model (see e.g. Azariadis, 1993, pp. 198-204) remain unaffected by the normalization.

6 Duffy and Papageorgiou (2000) use panel data techniques to estimate a cross-country aggregate CES production specification. For their entire sample of 82 countries they find that the elasticity of substitution between capital and (skilled) labor is significantly greater than unity.

7 In constructing Figure 4, we once again set $\bar{m}=1, \bar{k}=5$. 


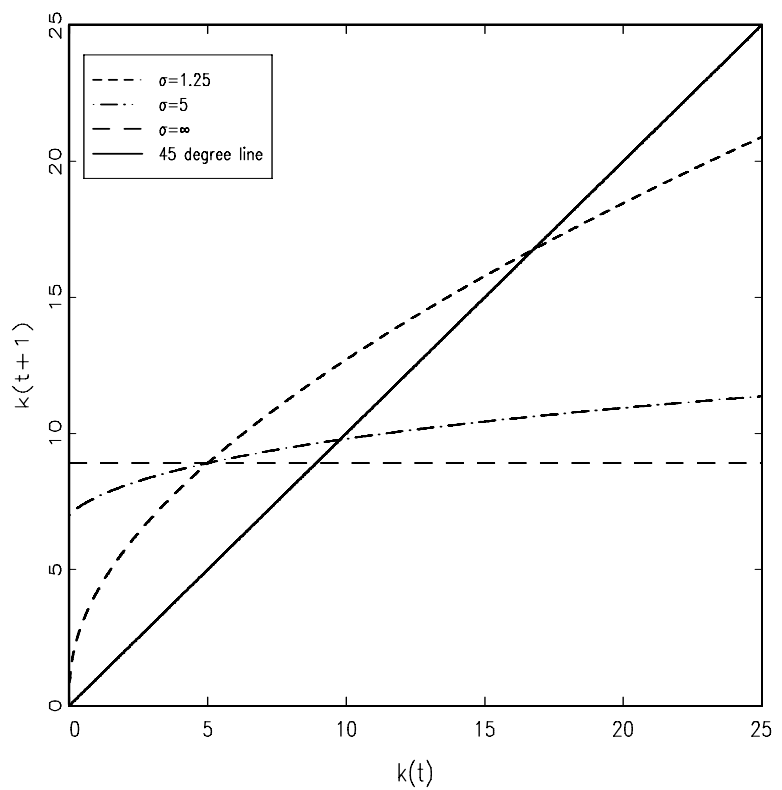

Figure 3. Transitional paths of per capita capital in the Diamond model when $\rho \geq \frac{\bar{m}}{\bar{k}}$

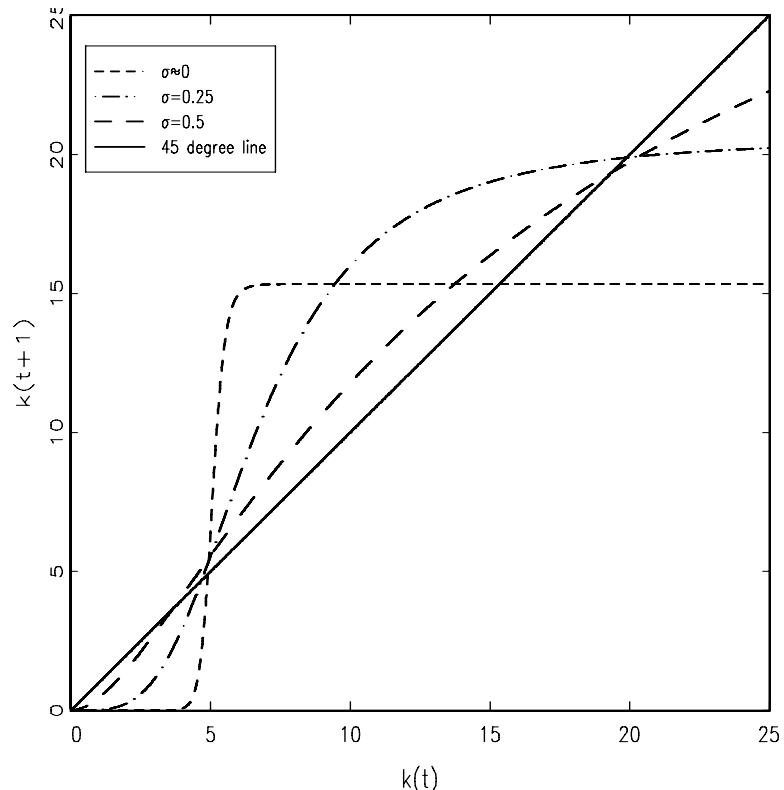

Figure 4. Transitional paths of per capita capital in the Diamond model when $\rho<\frac{\bar{m}}{k}$ 
come out of wage income in the former and out of total (wage and rental) income in the latter. A useful way to demonstrate the difference is offered by Galor (1996). Suppose that the fraction saved out of wage income, $\gamma^{w}$, differs from the fraction saved out of rental income, $\gamma^{r}$, possibly because of differences in preferences or endowments among agents. Then the law of motion for capital per worker in the normalized CES production function is

$$
k_{t+1}=\frac{\gamma^{w}}{1+n}\left[f_{\sigma}\left(k_{t}\right)-f_{\sigma}^{\prime}\left(k_{t}\right) k_{t}\right]+\frac{\gamma^{r}}{1+n} f_{\sigma}^{\prime}\left(k_{t}\right) k_{t} .
$$

Since $\gamma^{w}=\gamma^{r}=\gamma$ in the Solow model while $\gamma^{w}=\gamma$ and $\gamma^{r}=0$ in the Diamond model, the dynamical path in the former contains the additional term, $\frac{\gamma}{1+n} f_{\sigma}^{\prime}\left(k_{t}\right) k_{t}$, that represents savings out of rental income.

Now, to get an intuition of our result differentiate equation (4) with respect to $\sigma$ to obtain:

$$
\begin{aligned}
\frac{\partial k_{t+1}}{\partial \sigma}= & \frac{\gamma^{w}}{1+n} \underbrace{\left[\left(1-\pi_{t}\right) \frac{\partial f_{\sigma}\left(k_{t}\right)}{\partial \sigma}-f_{\sigma}\left(k_{t}\right) \frac{\partial \pi_{t}}{\partial \sigma}\right]}_{\frac{\partial w_{t}}{\partial \sigma}(?)} \\
& +\frac{\gamma^{r}}{1+n} \underbrace{\left[\pi_{t} \frac{\partial f_{\sigma}\left(k_{t}\right)}{\partial \sigma}+f_{\sigma}\left(k_{t}\right) \frac{\partial \pi_{t}}{\partial \sigma}\right]}_{\frac{\partial r k_{t}}{\partial \sigma}(+)},
\end{aligned}
$$

where $\pi_{t} \equiv \frac{f_{\sigma}^{\prime}\left(k_{t}\right) k_{t}}{f_{\sigma}\left(k_{t}\right)}$ is the rental income share. The first (second) expression on the right shows how wage (rental) income is affected by a change in $\sigma$. As shown by $\mathrm{KG}$, a higher elasticity of substitution implies an increase in income and rental shares. That makes the effect on rental income clearly positive since capital benefits from an increase in both output and rental share. But the effect on wage income is ambiguous as labor share falls. In the Solow model where total savings matter, however, those income distribution effects cancel out, leading to $\frac{\partial k_{t+1}}{\partial \sigma}>0$ as shown by KG.

In contrast, in the Diamond model where agents save a fraction of wage income, the second expression in (6) is absent. Within the first expression, $\left(1-\pi_{t}\right) \frac{\partial f_{\sigma}\left(k_{t}\right)}{\partial \sigma}$ measures how wage income increases when total output is increased, and this magnitude clearly depends on labor share, $\left(1-\pi_{t}\right)$. The second term, $-f_{\sigma}\left(k_{t}\right) \frac{\partial \pi_{t}}{\partial \sigma}$ represents a fall in labor share triggered by an increase in substitutability of capital for labor. If labor share $\left(1-\pi_{t}\right)$ is sufficiently small, the second term dominates the first, and our result follows. Indeed $\rho \geq \frac{\bar{m}}{\bar{k}}$ implies $(1-\bar{\pi})<1 / 2$ which is sufficient to obtain our result. ${ }^{8}$

\section{Conclusion}

In this paper we have shown that the positive relationship between the elasticity of substitution and economic growth discovered recently by KG (2000) does not

\footnotetext{
${ }^{8}$ Since $\bar{\pi}=\frac{\bar{k}}{k+\bar{m}}$ implies that $\frac{\bar{m}}{\bar{k}} \equiv \frac{1-\bar{\pi}}{\bar{\pi}}$; substitution into $\rho \geq \frac{\bar{m}}{\bar{k}}$ yields $\bar{\pi} \geq \frac{1}{1+\rho}$. Given that $\rho \in(0,1]$ under $\rho \geq \frac{\bar{m}}{\bar{k}}, \rho \geq \frac{\bar{m}}{\bar{k}}$ finally implies $\bar{\pi} \geq 1 / 2$.
} 
carry over to the Diamond model. Thus, whether the elasticity of substitution has a positive or negative effect on economic growth depends on our view of the world, that is, on the particular framework (Solow vs. Diamond) we believe to be a better representation of the world.

\section{Appendix}

Proof of Theorem 1. Rewrite equation (2) as

$$
\begin{aligned}
k_{t+1} & =\frac{\gamma}{1+n}\left[f_{\sigma}\left(k_{t}\right)-k_{t} f_{\sigma}^{\prime}\left(k_{t}\right)\right] \\
& =\frac{\gamma}{1+n}\left[f_{\sigma}\left(k_{t}\right)\left(1-\pi_{t}\right)\right],
\end{aligned}
$$

where $\pi_{t} \equiv \frac{f_{\sigma}^{\prime}\left(k_{t}\right) k_{t}}{f_{\sigma}\left(k_{t}\right)}$ is the rental income share. Differentiating with respect to $\sigma$ yields

$$
\frac{\partial k_{t+1}}{\partial \sigma}=\frac{\gamma}{1+n}\left[\left(1-\pi_{t}\right) \frac{\partial f_{\sigma}\left(k_{t}\right)}{\partial \sigma}-f_{\sigma}\left(k_{t}\right) \frac{\partial \pi_{t}}{\partial \sigma}\right] .
$$

Substituting $\frac{\partial f_{\sigma}\left(k_{t}\right)}{\partial \sigma}=-\frac{1}{\sigma^{2}} \frac{1}{\rho^{2}} y_{t}\left[\pi_{t} \ln \frac{\bar{\pi}}{\pi_{t}}+\left(1-\pi_{t}\right) \ln \frac{1-\bar{\pi}}{1-\pi_{t}}\right]$ and $\frac{\partial \pi_{t}}{\partial \sigma}=\frac{1}{\sigma^{2}}(1-$ $\left.\pi_{t}\right) \pi_{t} \ln \frac{k_{t}}{k}$ from Klump and de La Grandville (2000, pp. 284-285) yields

$$
\begin{aligned}
\frac{\partial k_{t+1}}{\partial \sigma}= & -\frac{\gamma}{1+n}\left\{\frac{\left(1-\pi_{t}\right) y_{t}}{\sigma^{2} \rho^{2}}\left[\pi_{t} \ln \frac{\bar{\pi}}{\pi_{t}}+\left(1-\pi_{t}\right) \ln \frac{1-\bar{\pi}}{1-\pi_{t}}\right]\right. \\
& \left.+\frac{y}{\sigma^{2}}\left(1-\pi_{t}\right) \pi_{t} \ln \frac{k_{t}}{\bar{k}}\right\} \\
= & -\frac{\gamma}{1+n} \frac{\left(1-\pi_{t}\right) y_{t}}{\sigma^{2} \rho^{2}} \\
& {\left[\pi_{t} \ln \frac{\bar{\pi}}{\pi_{t}}+\left(1-\pi_{t}\right) \ln \frac{1-\bar{\pi}}{1-\pi_{t}}+\rho^{2} \pi_{t} \ln \frac{k_{t}}{\bar{k}}\right], }
\end{aligned}
$$

where $\bar{\pi}=\frac{\bar{k}}{k+\bar{m}}$. From

$$
\begin{aligned}
& \frac{\bar{\pi}}{\pi_{t}}=\left(\frac{\bar{k}}{k_{t}} \frac{y_{t}}{\bar{y}}\right)^{\rho}, \\
& \frac{1-\bar{\pi}}{1-\pi_{t}}=\left(\frac{y_{t}}{\bar{y}}\right)^{\rho},
\end{aligned}
$$

we obtain $\frac{k_{t}}{k}=\frac{\bar{\pi}}{\pi_{t}} \frac{1-\pi_{t}}{1-\bar{\pi}}$. Substituting this into the last term in the brackets of equation (A1) gives

$$
\begin{aligned}
\frac{\partial k_{t+1}}{\partial \sigma}= & -\frac{\gamma}{1+n} \frac{\left(1-\pi_{t}\right) y_{t}}{\sigma^{2} \rho^{2}} \\
& {\left[\pi_{t} \ln \frac{\bar{\pi}}{\pi_{t}}+\left(1-\pi_{t}\right) \ln \frac{1-\bar{\pi}}{1-\pi_{t}}-\rho \pi_{t} \ln \left(\frac{\bar{\pi}}{\pi_{t}} \frac{1-\pi_{t}}{1-\bar{\pi}}\right)\right] . }
\end{aligned}
$$


Since the logarithmic function is strictly concave, we have that

$$
\begin{aligned}
\ln \frac{\pi_{t}}{\bar{\pi}}<\frac{\pi_{t}}{\bar{\pi}}-1 & \Rightarrow-\ln \frac{\pi_{t}}{\bar{\pi}}>1-\frac{\pi_{t}}{\bar{\pi}} \\
& \Rightarrow \ln \frac{\bar{\pi}}{\pi_{t}}>1-\frac{\pi_{t}}{\bar{\pi}} \\
\ln \frac{1-\pi_{t}}{1-\bar{\pi}}<\frac{1-\pi_{t}}{1-\bar{\pi}}-1 & \Rightarrow-\ln \frac{1-\pi_{t}}{1-\bar{\pi}}>1-\frac{1-\pi_{t}}{1-\bar{\pi}} \\
& \Rightarrow \ln \frac{1-\bar{\pi}}{1-\pi_{t}}>1-\frac{1-\pi_{t}}{1-\bar{\pi}}, \\
\ln \left(\frac{\bar{\pi}}{\pi_{t}} \frac{1-\pi_{t}}{1-\bar{\pi}}\right)<\frac{\bar{\pi}}{\pi_{t}} \frac{1-\pi_{t}}{1-\bar{\pi}}-1 & \Rightarrow-\ln \left(\frac{\bar{\pi}}{\pi_{t}} \frac{1-\pi_{t}}{1-\bar{\pi}}\right) \\
& >1-\frac{\bar{\pi}}{\pi_{t}} \frac{1-\pi_{t}}{1-\bar{\pi}} .
\end{aligned}
$$

Assume that $\sigma>1(\rho \in(0,1])$ and $k_{t}>\bar{k}$. Multiplying both sides of the final inequalities in (A2), (A3) and (A4) by $\pi_{t},\left(1-\pi_{t}\right)$ and $\rho \pi_{t}$, respectively, yields the following inequality:

$$
\begin{aligned}
& \pi_{t} \ln \frac{\bar{\pi}}{\pi_{t}}+\left(1-\pi_{t}\right) \ln \frac{1-\bar{\pi}}{1-\pi_{t}}-\rho \pi_{t} \ln \left(\frac{\bar{\pi}}{\pi_{t}} \frac{1-\pi_{t}}{1-\bar{\pi}}\right) \\
> & \pi_{t}\left(1-\frac{\pi_{t}}{\bar{\pi}}\right)+\left(1-\pi_{t}\right)\left(1-\frac{1-\pi_{t}}{1-\bar{\pi}}\right)+\rho \pi_{t}\left(1-\frac{\bar{\pi}}{\pi_{t}} \frac{1-\pi_{t}}{1-\bar{\pi}}\right) \\
= & \frac{\pi_{t}}{\bar{\pi}}\left(\bar{\pi}-\pi_{t}\right)+\frac{1-\pi_{t}}{1-\bar{\pi}}\left(\pi_{t}-\bar{\pi}\right)+\frac{\rho}{1-\bar{\pi}}\left(\pi_{t}-\bar{\pi}\right) \\
= & \left(\pi_{t}-\bar{\pi}\right)\left(\frac{1-\pi_{t}}{1-\bar{\pi}}+\frac{\rho}{1-\bar{\pi}}-\frac{\pi_{t}}{\bar{\pi}}\right) \\
= & \left(\frac{\pi_{t}-\bar{\pi}}{1-\bar{\pi}}\right)\left(1-\pi_{t}+\rho-\frac{\pi_{t}(1-\bar{\pi})}{\bar{\pi}}\right) \\
= & \left(\frac{\pi_{t}-\bar{\pi}}{1-\bar{\pi}}\right)\left(1+\rho-\frac{\pi_{t}}{\bar{\pi}}\right) \\
= & \left(\frac{\pi_{t}-\bar{\pi}}{1-\bar{\pi}}\right)\left[1+\rho-\left(\frac{\bar{k}+\bar{m}}{\bar{k}}\right) \frac{k_{t}^{\rho} \bar{k}^{1-\rho}}{k_{t}^{\rho} \bar{k}^{1-\rho}+\bar{m}}\right],
\end{aligned}
$$

where the last equality comes from the fact that $\pi_{t}=\frac{k_{t}^{\rho} \bar{k}^{1-\rho}}{k_{t}^{\rho} \bar{k}^{1-\rho}+\bar{m}}$. The function

$$
\phi\left(k_{t}\right) \equiv\left[1+\rho-\left(\frac{\bar{k}+\bar{m}}{\bar{k}}\right) \frac{k_{t}^{\rho} \bar{k}^{1-\rho}}{k_{t}^{\rho} \bar{k}^{1-\rho}+\bar{m}}\right],
$$

is monotonically decreasing with the horizontal asymptote at $\rho-\frac{\bar{m}}{\bar{k}}$. Therefore, if $\rho \geq \frac{\bar{m}}{\bar{k}}, \phi\left(k_{t}\right) \geq 0$. Then since $\frac{\pi_{t}-\bar{\pi}}{1-\bar{\pi}} \geq 0$ for $k_{t}>\bar{k}$, the last expression in (A5) is non-negative. Consequently, $\frac{\partial k_{t+1}}{\partial \sigma}<0$.

To prove that output per worker is a decreasing function of the $\sigma$ when $\rho \geq \frac{\bar{m}}{\bar{k}}$ and $k_{t}>\bar{k}$, rewrite $\frac{\partial y_{t+1}}{\partial \sigma}$ as

$$
\frac{\partial y_{t+1}}{\partial \sigma}=\frac{\partial y_{t+1}}{\partial k_{t+1}} \frac{\partial k_{t+1}}{\partial \sigma} .
$$


We have just shown that $\frac{\partial k_{t+1}}{\partial \sigma}<0$ for $\rho \geq \frac{\bar{m}}{k}$ and $k_{t}>\bar{k}$. Given that $\frac{\partial y_{t+1}}{\partial k_{t+1}}$ is positive for all $k_{t+1}>0$, then $\frac{\partial y_{t+1}}{\partial \sigma}<0$. This completes the proof of Theorem 1A.

To prove Theorem 1B, define the growth rate of capital per worker by $g_{k}=$ $\frac{k_{t+1}}{k_{t}}-1$ and the growth rate of output per worker by $g_{y}=\frac{y_{t+1}}{y_{t}}-1$. Differentiation yields

$$
\begin{gathered}
\frac{\partial g_{k}}{\partial \sigma}=\frac{1}{k_{t}} \frac{\partial k_{t+1}}{\partial \sigma}<0, \forall \rho \geq \frac{\bar{m}}{\bar{k}}, \text { and } k_{t}>\bar{k}, \\
\frac{\partial_{y}}{\partial \sigma}=\frac{1}{y_{t}} \frac{\partial y_{t+1}}{\partial \sigma}<0, \forall \rho \geq \frac{\bar{m}}{\bar{k}}, \text { and } k_{t}>\bar{k} .
\end{gathered}
$$

This completes the proof.

Proof of Theorem 2. At steady state, $k_{t}=k_{t+1}=k^{*}$ and therefore equation (2) reduces to the polynomial equation (3). Differentiating $k^{*}$ with respect to $\sigma$ yields

$$
\frac{\partial k_{\sigma}^{*}}{\partial \sigma}=\frac{\frac{\gamma}{1+n}\left(w_{\sigma}^{*}\right)^{\prime}}{1-\frac{\gamma}{1+n}\left(w_{k}^{*}\right)^{\prime}},
$$

where $\left(w_{\sigma}^{*}\right)^{\prime}=\left[\left(1-\pi^{*}\right) \frac{\partial f^{*}}{\partial \sigma}-f^{*} \frac{\partial \pi^{*}}{\partial \sigma}\right]$ and $\left(w_{k}^{*}\right)^{\prime}=\left[\left(1-\pi^{*}\right)\left(f^{*}\right)^{\prime}-f^{*} \frac{\partial \pi^{*}}{\partial k^{*}}\right]$. By Theorem 1, $\left(w_{\sigma}^{*}\right)^{\prime}<0$ for $\rho \geq \frac{\bar{m}}{k}$ and $k^{*}>k_{t}$, so the numerator is negative. To show that the denominator is positive, solve the definition of $\pi^{*}=\frac{\left(f^{*}\right)^{\prime} k^{*}}{f^{*}}$ and the steady-state polynomial equation $k^{*}=\frac{\gamma}{1+n}\left[f^{*}-\left(f^{*}\right)^{\prime} k^{*}\right]$ simultaneously for $f^{*}$ and $\left(f^{*}\right)^{\prime}$ to obtain

$$
\begin{gathered}
f^{*}=\frac{(1+n)}{\gamma} \frac{k^{*}}{1-\pi^{*}}, \\
\left(f^{*}\right)^{\prime}=\frac{(1+n)}{\gamma} \frac{\pi^{*}}{1-\pi^{*}} .
\end{gathered}
$$

Substituting equations (B2), (B3) and $\frac{\partial \pi^{*}}{\partial k^{*}}=\frac{\rho \pi^{*}\left(1-\pi^{*}\right)}{k^{*}}$ into $\left(w_{k}^{*}\right)^{\prime}$ gives

$$
\begin{aligned}
\left(w_{k}^{*}\right)^{\prime} & =\left(1-\pi^{*}\right) \frac{(1+n)}{\gamma} \frac{\pi^{*}}{1-\pi^{*}}-\frac{(1+n)}{\gamma} \frac{k^{*}}{1-\pi^{*}} \frac{\rho \pi^{*}\left(1-\pi^{*}\right)}{k^{*}} \\
& =\frac{(1+n)}{\gamma}(1-\rho) \pi^{*} .
\end{aligned}
$$

Then $\frac{\gamma}{1+n}\left(w_{k}^{*}\right)^{\prime}=(1-\rho) \pi^{*}$ and hence $1-\frac{\gamma}{1+n}\left(w_{k}^{*}\right)^{\prime}=1-(1-\rho) \pi^{*}>0$. Therefore $\frac{\partial k^{*}}{\partial \sigma}<0$.

To prove that the steady-state output per worker is a decreasing function of the $\sigma$ when $\rho \geq \frac{\bar{m}}{k}$ and $k_{t}>\bar{k}$, once again rewrite $\frac{\partial y^{*}}{\partial \sigma}$ as

$$
\frac{\partial y^{*}}{\partial \sigma}=\frac{\partial y^{*}}{\partial k^{*}} \frac{\partial k^{*}}{\partial \sigma} .
$$

Given that $\frac{\partial y^{*}}{\partial k^{*}}>0$ for all $k^{*}>0$, and $\frac{\partial k^{*}}{\partial \sigma}<0$ for $\rho \geq \frac{\bar{m}}{k}$ and $k_{t}>\bar{k}$ as shown above, $\frac{\partial y^{*}}{\partial \sigma}<0$ as desired. 


\section{References}

Arrow, K. J., Chenery, H. B., Minhas, B.S., Solow, R. M.: Capital-labor substitution and economic efficiency. Review of Economics and Statistics 43, 225-250 (1961)

Azariadis, C.: Intertemporal macroeconomics. Cambridge, MA: Blackwell Publishers 1993

Diamond, P. A.: National debt in a neoclassical growth model. American Economic Review 55, 1126$1150(1965)$

Duffy, J., Papageorgiou, C.: A cross-country empirical investigation of the aggregate production function specification. Journal of Economic Growth 5, 87-120 (2)

Galor, O.: Convergence? Inferences from theoretical models. Economic Journal 106, 1056-1069 (1996)

Hicks, J. R.: The theory of wages. London: MacMillan 1932

Klump, R., de La Grandville, O.: Economic growth and elasticity of substitution: Two theorems and some suggestions. American Economic Review 90, 282-291 (2000)

Klump, R., Preissler, H.: CES production functions and economic growth. Scandinavian Journal of Economics 102, 41-56 (2000)

Pitchford, J. D.: Growth and the elasticity of substitution. Economic Record 36, 491-504 (1960)

Sato, R.: The most general class of CES functions. Econometrica 43, 999-1003 (1975)

Shimomura, K.: A simple proof of the Sato proposition on non-homothetic CES functions. Economic Theory 14, 501-503 (1999)

Solow, R. M.: A contribution to the theory of economic growth. Quarterly Journal of Economics 70, 65-94 (1956) 